EPJ Web of Conferences 76, 01021 (2014)

DOI: $10.1051 /$ epjconf/20147601021

(C) Owned by the authors, published by EDP Sciences, 2014

\title{
Thin helical vortex dynamics in low-viscosity liquid
}

\author{
M.V. Agafontseva ${ }^{2}$ and P.A. Kuibin ${ }^{1,2, a}$ \\ ${ }^{1}$ Novosibirsk State University, Russia \\ ${ }^{2}$ Kutateladze Institute of Thermophysics, Novosibirsk, Russia
}

\begin{abstract}
The problem of helical vortices description has the significant interest as from fundamental point of view as well for practice. In some sense this problem is close to the vortex ring one which attracted much more attention in last decades. The reviews on the vortex rings investigations can be found in recent papers [1,2] or in book by Akhmetov [3]. In particular, in series of papers by Kaplanskii with co-authors [4-6] there was considered the viscosity influence on the vortex ring evolution. Separate attention was paid to the low Reynolds number case and to high Reynolds number one, initial stage of viscous evolution and final one. This paper presents first attempt for research on the diffusion and dynamics of a viscous helical vortex.
\end{abstract}

In this work the problem of thin helical dynamics in low-viscosity liquid is considered. Appearance of helical vortex in the flow assumes helical symmetry. It means, that all of flow characteristic will be constant along the helical lines.

The flow of viscous incompressible fluid under condition of the helical symmetry is described in variables vorticity - stream function by the system of equations $[7,8]$

$$
\begin{gathered}
\frac{\partial^{2} \psi}{\partial r^{2}}+\left(1-\frac{2 r^{2}}{r^{2}+l^{2}}\right) \frac{1}{r} \frac{\partial \psi}{\partial r}+\frac{r^{2}+l^{2}}{r^{2} l^{2}} \frac{\partial^{2} \psi}{\partial \chi^{2}}=2 u_{0} \frac{l}{r^{2}+l^{2}}-\omega_{z} \frac{r^{2}+l^{2}}{l^{2}} \\
\frac{\partial \omega_{z}}{\partial t}+\frac{1}{r} \frac{\partial \psi}{\partial \chi} \frac{\partial \omega_{z}}{\partial r}-\frac{1}{r} \frac{\partial \psi}{\partial r} \frac{\partial \omega_{z}}{\partial \chi}=v \Delta \omega_{z} .
\end{gathered}
$$

Here $(\mathrm{r}, \chi)$ is helical variables, where $\chi$ is determined with axial and tangential coordinates of cylindrical system: $\chi=\theta-z / l,(\mathrm{r}, \theta, \mathrm{z}), 2 \pi \mathrm{l}$ is the pitch of helical symmetry, $\omega \mathrm{z}$ is the axial component of vorticity vector, $\psi$ is the stream function which relates to the velocity components as (3)

$$
\frac{\partial\left(r u_{r}\right)}{\partial r}+\frac{\partial u_{\chi}}{\partial \chi}=0
$$

Cartesian equation of continuity is

$$
\frac{\partial\left(u_{x}\left(y^{2}+l^{2}\right)-x y \cdot u_{y}\right)}{\partial x}+\frac{\partial\left(u_{y}\left(x^{2}+l^{2}\right)-x y \cdot u_{x}\right)}{\partial y}=0
$$

\footnotetext{
${ }^{a}$ Corresponding author: kuibin@itp.nsc.ru
}

This is an Open Access article distributed under the terms of the Creative Commons Attribution License 4.0, which permits unrestricted use, distribution, and reproduction in any medium, provided the original work is properly cited. 

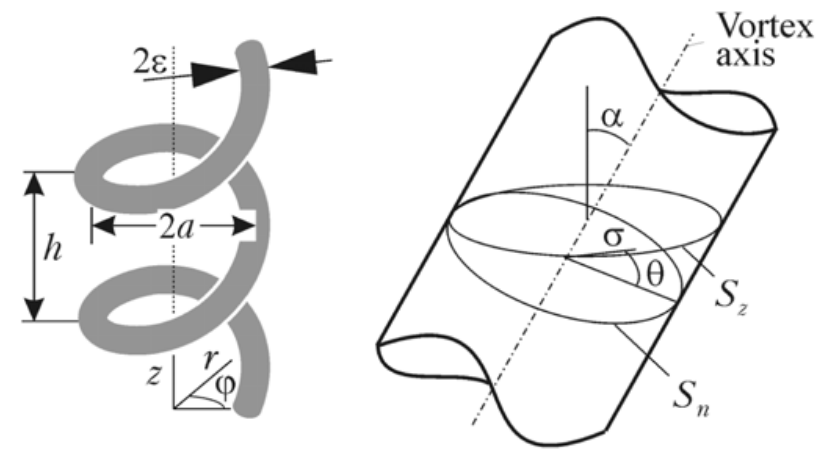

Figure 1. Scheme of helical vortex. $\mathrm{h}=2 \pi \mathrm{l}, \mathrm{S}_{\mathrm{z}}$ is the vortex core cross-section by plane $\mathrm{z}=$ const, $\mathrm{S}_{\mathrm{n}}$ is its normal cross-section.

Stream function is determined with follows correlations:

$$
\begin{aligned}
u_{x}\left(y^{2}+l^{2}\right)-x y \cdot u_{y} & =\frac{\partial \psi}{\partial y}, \quad u_{y}\left(x^{2}+l^{2}\right)-x y \cdot u_{x}=-\frac{\partial \psi}{\partial x}, \text { or } \\
u_{x} & =\frac{1}{l^{2}\left(x^{2}+y^{2}+l^{2}\right)}\left(\left(x^{2}+l^{2}\right) \frac{\partial \psi}{\partial y}-x y \frac{\partial \psi}{\partial x}\right), \\
u_{y} & =\frac{1}{l^{2}\left(x^{2}+y^{2}+l^{2}\right)}\left(x y \frac{\partial \psi}{\partial y}-\left(y^{2}+l^{2}\right) \frac{\partial \psi}{\partial x}\right) .
\end{aligned}
$$

Now we define Cartesian vorticity vector components taking to account the flow helical symmetry $\left(u_{z}=u_{0}+\frac{y}{l} u_{x}-\frac{x}{l} u_{y}, \frac{\partial}{\partial z}=\frac{y}{l} \frac{\partial}{\partial x}-\frac{x}{l} \frac{\partial}{\partial y}\right):$

$$
\omega_{x}=\frac{u_{x}-y \omega_{z}}{l}, \quad \omega_{y}=\frac{u_{y}+x \omega_{z}}{l}, \quad \omega_{z}=\frac{\partial u_{y}}{\partial x}-\frac{\partial u_{x}}{\partial y} .
$$

Boundary conditions for helical vortex:

$$
\psi(0,0, z)=\omega_{z}(0,0, z)=0 ; \quad x \rightarrow \infty, \quad y \rightarrow \infty: \quad \psi \rightarrow 0, \quad \omega_{z} \rightarrow 0 .
$$

Expressions given above refer to the case of the right helical symmetry flow. In the case of the left helical symmetry flow we should to account variable 1 to be a positive. For the left symmetry transition it is easily to change the sign of 1 ( 1 will be negative).

There are some methods of solving of the helical vortex diffusion. One of these methods is to fix variable 1 and to find the solution as a function which depends on the vortex core radius $\varepsilon$ and the vortex radius a. (see Fig. 1).

Let us find the solution of the Eqs. ((1)-(2)) for the helical vortex with thin core which spreading in the low-viscosity liquid. As in the paper [5], we set some initial non dimensional values of the helical radius and helical core radius: $a_{*}\left(t_{0}\right)=a_{0}, \quad \varepsilon_{*}\left(t_{0}\right)=\varepsilon_{0}$.

After that, we introduce the local coordinate $(\sigma, \theta)$ in a plane perpendicular to the vortex axis and cross the axis in the point $(\mathrm{a}, 0,0)$

$$
\begin{aligned}
& \sqrt{x^{2}+y^{2}} \cos (\phi)=a+\sigma \cos (\theta), \\
& \sqrt{x^{2}+y^{2}} \sin (\phi)=\sigma \sin (\theta) \cos (\alpha), \\
& z=-\sigma \sin (\theta) \sin (\alpha),
\end{aligned}
$$


and we consider two types of the non dimensional:

$$
\begin{gathered}
\sigma^{*}=\frac{\sigma}{a_{*}}, \quad \tau^{*}=\frac{a_{0}}{a_{*}} \\
\bar{\sigma}=\frac{\sigma}{\varepsilon_{*}}, \quad \bar{\tau}=\frac{\varepsilon_{0}}{\varepsilon_{*}} .
\end{gathered}
$$

Unknown functions is presented as a sum of two members, each of them depends on one of variable type:

$$
\begin{aligned}
& \frac{\omega\left(\sigma, \theta, t ; \delta_{0}\right)}{\omega_{0}}=\omega^{*}\left(\sigma^{*}, \theta^{*}, \tau^{*} ; \delta_{0}\right)+\frac{\bar{\omega}\left(\bar{\sigma}, \bar{\theta}, \bar{\tau} ; \delta_{0}\right)}{\delta_{0}^{2}} \\
& \frac{\psi\left(\sigma, \theta, t ; \delta_{0}\right)}{\omega_{0} a_{*}^{3}}=\psi^{*}\left(\sigma^{*}, \theta^{*}, \tau^{*} ; \delta_{0}\right)+\bar{\psi}\left(\bar{\sigma}, \bar{\theta}, \bar{\tau} ; \delta_{0}\right) .
\end{aligned}
$$

Here $\omega_{0}$ is the some of characteristic vorticity scale is determined separately, $\delta_{0}=\varepsilon_{0} / \mathrm{a}_{0}$ is the decomposition parameter with the same order of the value $\delta=\varepsilon^{*} / \mathrm{a}^{*}$.

The zero-th order solution for vortex diffusion that is valid as for rectilinear vortex as well as for any curved vortex is obviously the Lamb-Oseen solution:

$$
\omega_{\tan }(\sigma, t)=\frac{\Gamma}{4 \pi v t} e^{-\sigma^{2} / 4 v t}
$$

$\omega$ tan denotes the vorticity projection on the tangent to the vortex axis.

Analysis of Eqs. ((1),(2)) in view of decomposition $(4,5)$ yields first approximation

$$
\bar{\omega}_{0}(\bar{\sigma}, \theta, \bar{\tau} ; 0)=\exp \left(-\frac{\bar{\sigma}^{2}+\bar{\tau}^{2}}{2}\right) I_{0}(\bar{\sigma} \bar{\tau})
$$

that has the same view as solution for vortex ring in [5]. Here I0 is the modified Bessel function of zero-th order.

Finally we have derived that at passing through a long time period the vorticity distribution tends again to the Lamb-Oseen solution

$$
\omega_{z}(r, t)=\frac{\Gamma}{4 \pi v t} e^{-r^{2} / 4 v t}
$$

As a result in this work the first results on the thin vortex diffusion at the high Reynolds numbers was obtained. In the future is planned to obtain as the vorticity and stream function members of the higher order as well as the helical vortex spread velocity depending on time and to consider the case of the low Reynolds numbers.

The work was supported by RFBR (project 11-08-01063), Program for support of leading scientific schools (project 6686.2012.8), Program "R\&D on priority directions of development of the scientific-technological complex of Russia" (contract 11.519.11.6022), and Program "Scientific and scientificpedagogical staff of innovation Russia" (grant 8225).

\section{References}

[1] F. Kaplanski, Y. Fukumoto, Y. Rudi, Physics of Fluids, 24 (2012)

[2] Y. Fukumoto, Theoretical and Computational Fluid Dynamics. 24 (2010) 


\section{EPJ Web of Conferences}

[3] D.G. Akhmetov, Vortex Rings, (2009)

[4] A.A. Berezovskii, F.B. Kaplanskii, Fluid Dynamics. 22 (1987)

[5] A.A. Berezovskii, F.B. Kaplanskii, Fluid Dynamics. 27 (1992)

[6] F.B. Kaplanskii, Yu.A. Rudi, Fluid Dynamics. 36 (2001)

[7] S.V. Alekseenko, P.A. Kuibin, V.L. Okulov, S.I. Shtork, J. Fluid Mech. 382 (1999)

[8] S.V. Alekseenko, P.A. Kuibin, V.L. Okulov, Theory of Concentrated Vortices an Introdution, (2007) 